\title{
Widely Tunable Picosecond Fiber Ring Laser Mode-locked by a Single-wall Carbon Nanotube (SWNT)-Polycarbonate Composite Film Saturable Absorber
}

\author{
F. Wang, R.V. Penty, I.H. White, Z. Sun, A. Rozhin, V. Scardaci and A. Ferrari, \\ Department of Engineering, University of Cambridge, 9 JJ Thomson Avenue, Cambridge, CB3 OFA, UK \\ Email address: ihw3@cam.ac.uk
}

\begin{abstract}
A widely tunable fiber ring laser, utilising a SWNT/polycarbonate film mode-locker and a 3 -nm tunable filter, has been realized. $2.3 \mathrm{ps}$ pulse generation over $27 \mathrm{~nm}$ spectral range is achieved for a constant pump power of $25 \mathrm{~mW}$.

(O2007 Optical Society of America

OCIS codes: $(140,3600)$ Lasers, tunable; $(140,4050)$ Mode-locked lasers; (230.4320) Nonlinear optical devices.
\end{abstract}

\section{Introduction}

Widely tunable ultrashort pulse sources are of particular importance for femtosecond science and related fields. A number of technologies have been employed for the realization of such sources [1-2]. Recently, single-wall carbon nanotubes (SWNTs) have emerged as a new saturable absorber material. They possess advantages such as subpicosecond recovery time [3-4], high optical damage threshold [5], and wide absorption bandwidth. These features make them well suited for wavelength tunable ultrashort mode-locked pulse generation. Although fiber lasers [6], waveguide lasers [7] and solid-state lasers [5] have all been successfully mode-locked using SWNTs or SWNTpolymer composites, there has been no investigation to date on the wavelength tunability of such SWNT-based mode-locked lasers.

In this paper, we demonstrate for the first time a tunable picosecond fiber ring laser mode-locked by a SWNTpolycarbonate (SWNT-PC) composite film. The laser produces $2.3 \mathrm{ps}$ transform-limited pulses at a repetition rate of 14.4 MHz. A 3-nm bandpass filter (operating wavelength $1530-1560 \mathrm{~nm}$ ) is utilized in the cavity as a wavelength selection element. The output wavelength can be continuously tuned from $1533 \mathrm{~nm}$ to $1560 \mathrm{~nm}$, a range of $27 \mathrm{~nm}$, with constant pulse duration and output power for a given drive current.

\section{Experimental Setup and Results}

The procedure to prepare the SWNT-PC composite film can be summarized as follows: SWNT powders are dispersed in dichlorobenzene (DCB) in presence of poly (3-hexylthiophene-2, 5-diyl) (P3HT) by ultrasonication. The solution is then filtered through a $1 \mu \mathrm{m}$ retention filter and centrifuged at $300,000 \mathrm{~g}$ to remove bundles and impurities. Pellets of polycarbonate are dissolved in the solution by ultrasonication. The final mixture is dried at room temperature under vacuum to form a film with a thickness typically of 20 microns or more. Fig. 1 shows the linear optical absorption measured in the visible and near IR spectral range and confirms the wide absorption band located near the laser's operating wavelength. The mode-locker is then formed by sandwiching the free-standing SWNT-PC film between two fibre ferrules inside a FC/PC connector [8].

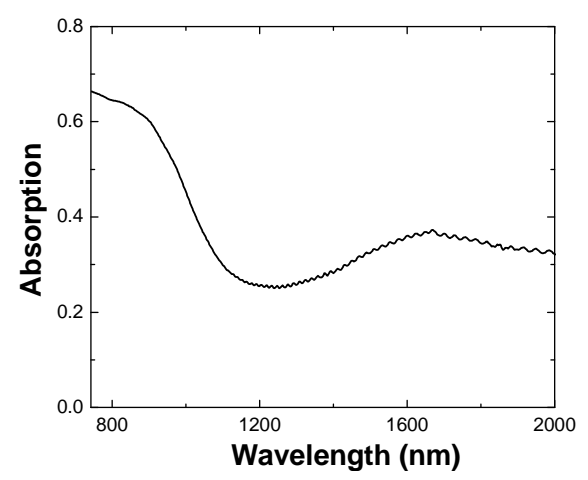

Fig.1. Linear absorption spectrum

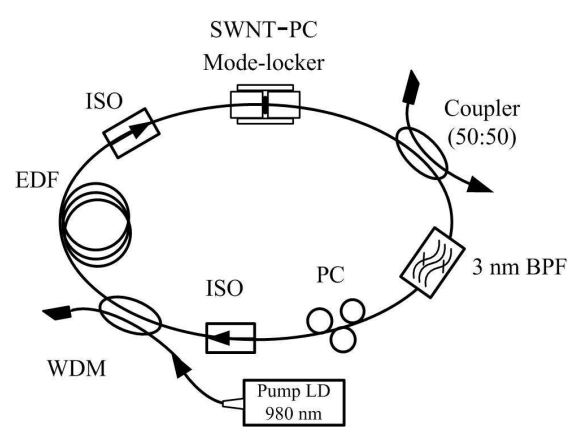

Fig. 2. Ring laser experimental setup. 


\section{JThA36.pdf}

The laser setup is shown in Fig. 2. A 1-metre span of highly-doped $\mathrm{Er}^{3+}$ fibre acts as the gain medium. It is pumped by a $980 \mathrm{~nm}$ diode laser via a WDM coupler. Two isolators were placed at both ends of the amplification section to maintain uni-directional laser operation. The SWNT-PC mode-locker is placed after the isolator at the output of the amplification section to enhance saturable absorption, and light is then coupled out of the cavity via a 50/50 coupler. A 3-nm bandpass filter (operating wavelength 1530-1560nm) following the output coupler acts as the wavelength tuning element. A polarization controller (PC) is used to optimize the mode-locking condition. The total length of the laser cavity is estimated to be around 13.9 metres.

At a pump power of $25 \mathrm{~mW}$, mode-locking self-starts and the laser produces a stable pulse train at a repetition rate of $14.43 \mathrm{MHz}$. By changing the central wavelength of the bandpass filter, the laser output can be tuned from 1533 $\mathrm{nm}$ to $1560 \mathrm{~nm}$ without the need to vary the pump power. Fig. 3(a) shows the lasing spectra and the associated background free autocorrelation traces. By way of comparison, it should be noted that without the tunable filter, the ring laser mode-locks at a centre wavelength of $1555 \mathrm{~nm}$ with a pulse width of 496fs.

As the bandpass filter is tuned, the laser wavelength may be varied from $1533-1560 \mathrm{~nm}$. A small side peak to the short wavelength side of the central peak is visible for all the operating wavelengths. This may be caused by the insufficient modulation index of the saturable absorber due to relatively low intracavity power level. Fig. 3(b) shows the corresponding autocorrelation traces at different output wavelengths. Fig. 4 shows the laser output power and pulse duration at different wavelengths. The traces overlap well with each other, indicating only a small fluctuation in the output pulse width as the wavelength is varied. The average output power across the C-band is $-5.3 \mathrm{dBm}$ with a power fluctuation smaller than $0.6 \mathrm{~dB}$. From Fig. 4 the average pulse duration for different wavelengths is $2.3 \mathrm{ps}$ assuming a sech ${ }^{2}$ temporal profile. This leads to a typical time-bandwidth product of 0.316 , demonstrating near chirp free, Fourier limited pulse generation.
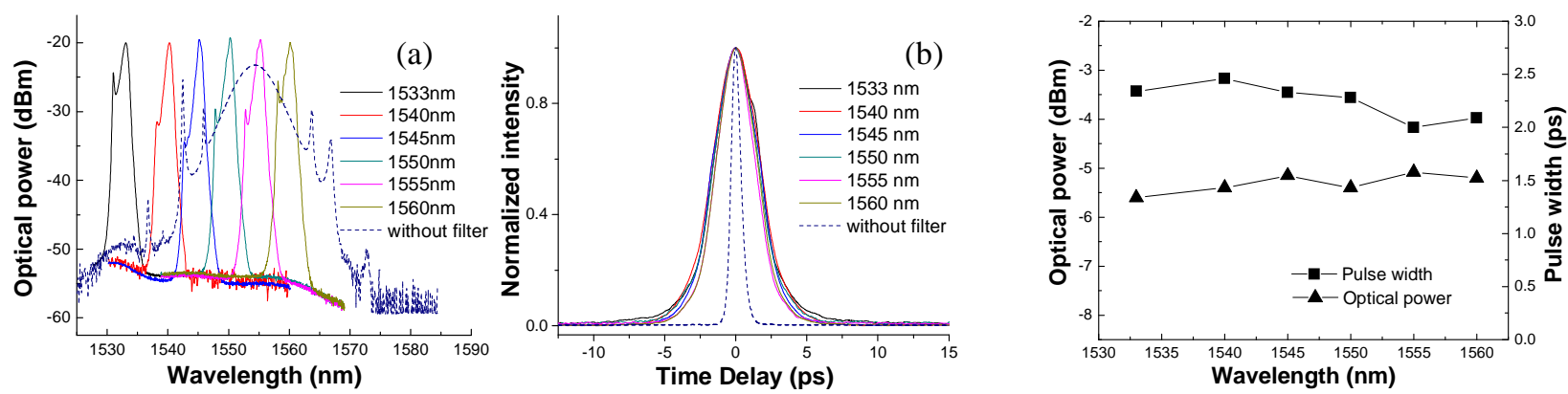

Fig.3. (a ) Optical spectra (b) Autocorrelation traces at different wavelengths ,(dotted: without filter).

Fig. 4. Output power and pulse widths as a function of laser operating wavelength.

\section{Conclusions}

In summary, we have successfully demonstrated a widely tunable picosecond pulsed laser employing a SWNTPolycarbonate composite film saturable absorber. The output wavelength can be continuously tuned from $1533 \mathrm{~nm}$ to $1560 \mathrm{~nm}$, with constant pulse duration and output power for a set drive current. By increasing the bandwidth of the tunable filter and the gain bandwith of the laser gain medium, shorter pulse duration and wider tuning range may be realized.

\section{Reference}

[1] D. Kopf et al., "Broadly tunable femtosecond Cr:LiSAF laser", Opt. Lett., 22, 621 (1997)

[2] F. Tauser et al., "Widely tunable sub-30-fs pulses from a compact erbium-doped fiber source", Opt. Lett., 29, 516 (2004)

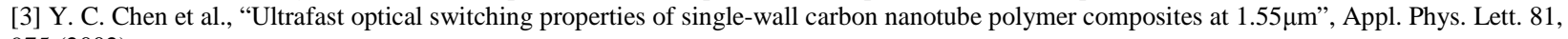
975 (2002).

[4] S. Tatsuura et al., "Semiconductor carbon nanotubes as ultrafast switching materials for optical communication ", Adv. Mater. 15, 534 (2003).

[5] K. H. Fong et al., "Solid-state Er:Yb:glass laser mode-locked by using single-wall carbon nanotube thin film", Opt. Lett. 32, 38 (2007).

[6] S. Y. Set et al., in Optical Fiber Communication Conference (OFC), Vol. 87 of OSA Trends in Optics and Photonics (Optical Society of America, 2003), postdeadline paper PD44.

[7] G. Della Valle et al., "Passive mode locking by carbon nanotubes in a femtosecond laser written waveguide laser", Appl. Phys. Lett. 89, 231115 (2006).

[8] A.G. Rozhin et al., "Generation of ultra-fast laser pulses using nanotube mode-lockers", Phys. Stat. Sol. (b) 243, 3551 (2006). 